Portland State University

PDXScholar

\title{
Enhanced magnetic quantum oscillations in the mixed state of a two-dimensional organic superconductor
}

\author{
J. Wosnitza \\ J. Hagel \\ P. J. Meeson \\ D. Bintley
}

J. A. Schlueter

See next page for additional authors

Follow this and additional works at: https://pdxscholar.library.pdx.edu/chem_fac

Part of the Atomic, Molecular and Optical Physics Commons, and the Materials Chemistry Commons Let us know how access to this document benefits you.

\section{Citation Details}

J. Wosnitza, J. Hagel, P.J. Meeson, J.A. Schlueter, J. Mohtasham and R. W. Winter, "Enhanced Magnetic Quantum Oscillations in the Mixed State of a Two-Dimensional Organic Superconductor," Phys. Rev. B, 67, 060504-1 (2003).

This Article is brought to you for free and open access. It has been accepted for inclusion in Chemistry Faculty Publications and Presentations by an authorized administrator of PDXScholar. Please contact us if we can make this document more accessible: pdxscholar@pdx.edu. 


\section{Authors}

J. Wosnitza, J. Hagel, P. J. Meeson, D. Bintley, J. A. Schlueter, Javid Mohtasham, Rolf Walter Winter, and Gary L. Gard 


\title{
Enhanced magnetic quantum oscillations in the mixed state of a two-dimensional organic superconductor
}

\author{
J. Wosnitza, ${ }^{1}$ J. Hagel, ${ }^{1,2}$ P. J. Meeson, ${ }^{3}$ D. Bintley, ${ }^{3}$ J. A. Schlueter, ${ }^{4}$ J. Mohtasham, ${ }^{5}$ R. W. Winter, ${ }^{5}$ and G. L. Gard ${ }^{5}$ \\ ${ }^{1}$ Institut für Angewandte Physik (IAPD), Technische Universität Dresden, D-01062 Dresden, Germany \\ ${ }^{2}$ Grenoble High Magnetic Field Laboratory, MPI and CNRS, 38042 Grenoble, France \\ ${ }^{3}$ H. H. Wills Physics Laboratory, University of Bristol, Bristol, BS8 1TL, United Kingdom \\ ${ }^{4}$ Materials Science Division, Argonne National Laboratory, Argonne, Illinois 60439 \\ ${ }^{5}$ Department of Chemistry, Portland State University, Portland, Oregon 97207
}

(Received 9 December 2002; published 28 February 2003)

\begin{abstract}
We report on de Haas-van Alphen (dHvA) oscillations observed in the mixed state of the organic superconductor $\beta^{\prime \prime}$-(BEDT-TTF) ${ }_{2} \mathrm{SF}_{5} \mathrm{CH}_{2} \mathrm{CF}_{2} \mathrm{SO}_{3}$ (BEDT-TTF stands for bisethylenedithio-tetrathiafulvalene) utilizing the field-modulation technique and torque magnetometry. At low temperatures $(30 \mathrm{mK})$, the $\mathrm{dHvA}$ signal persists down to $1.4 \mathrm{~T}$ well below the upper critical field $B_{c 2} \approx 3.6 \mathrm{~T}$. Contrary to most theoretical predictions and previous experimental findings, no additional damping of the dHvA-oscillation amplitude, but a reduced damping of the dHvA signal is found. This highly unusual effect might indicate a reduced quasiparticle scattering rate or an additional oscillatory contribution in the superconducting state.
\end{abstract}

DOI: 10.1103/PhysRevB.67.060504

PACS number(s): 74.70.Kn, 71.18.+y, 74.25.Jb

The occurrence of de Haas-van Alphen (dHvA) oscillations deep in the mixed state seems to be a universal property for type-II superconductors. This has been confirmed experimentally for a large number of materials in recent years. ${ }^{1}$ On first sight, these observations are very puzzling since the evolution of the superconducting energy gap should quickly suppress any magnetic quantum oscillations which usually imply the existence of a Fermi surface. Although the microscopic mechanism resulting in magnetic quantum oscillations in the mixed state is still an open question, all theories dealing with this subject agree on the central role played by the energy gap. ${ }^{1,2}$ Most of these theories predict an additional attenuation of the dHvA signal in the superconducting state. For superconductors with anisotropic gap or even gap nodes, the angular dependence of this attenuation would yield valuable information on the magnitude and angular variation of the energy gap. ${ }^{3}$

In the normal state, the usual damping of the dHvA oscillations is caused by quasiparticle scattering with rate $\tau_{0}^{-1}$ due to static defects and impurities. This is described by the Dingle damping factor $R_{D}=\exp \left(-r \pi m_{b} / e B \tau_{0}\right)$, with $r$ counting the oscillation harmonics and $m_{b}$ is the band mass averaged around the cyclotron orbit and unaffected by manybody interactions. ${ }^{1,4}$ Instead of $\tau_{0}$, the Dingle temperature $T_{D}=\hbar / 2 \pi k_{B} \tau_{0}$ is often used. The additional damping of the dHvA signal towards lower $B$ in the mixed state can be described in analogy to $R_{D}$ by a damping factor $R_{S}$ $=\exp \left(-r \pi m_{b} / e B \tau_{s}\right)$, where $\tau_{s}$ governs the field, temperature, and spatial variation of the superconducting order parameter. The various theoretical models predict different dependences. However, no consistent explanation of the experimentally observed dHvA signals in the mixed state has been achieved yet.

We recently reported about the fundamentally different phenomenon of Shubnikov-de Haas (SdH) oscillations in the superconducting vortex-liquid state. $^{2}$ For the strongly type-II organic superconductor $\beta^{\prime \prime}$-(BEDT-TTF $)_{2} \mathrm{SF}_{5} \mathrm{CH}_{2} \mathrm{CF}_{2} \mathrm{SO}_{3}\left(T_{c} \approx 4.4 \mathrm{~K},{ }^{5}\right.$ BEDT-TTF is equivalent to ET which stands for bisethylenedithiotetrathiafulvalene), we were able to observe dHvA and $\mathrm{SdH}$ oscillations down to about $1.7 \mathrm{~T}$ at $50 \mathrm{mK}$, which is well below the upper critical field, $B_{c 2} \approx 3.6 \mathrm{~T}$, and well below the irreversibility field $B_{i r r}$ as proven by magnetization data (see below). Contrary to the usually found and theoretically predicted behavior, no additional damping of the $\mathrm{SdH}$ as well as of the dHvA signal-extracted from magnetization data measured by use of the torque method-was observed in the mixed state below $B_{c 2}$ and $B_{i r r} .{ }^{2}$ There was even an indication that the measured quantum-oscillation amplitude in the mixed state occurred to be somewhat enlarged as compared to the conventional normal-state behavior.

To study this unique behavior in more detail, we performed additional dHvA measurements by means of the torque method and, further on, utilized a different technique, namely, the field-modulation method which, for the present case, is especially sensitive at low magnetic fields. Both sets of experiments were performed in top-loading dilution refrigerators allowing to reach some $10 \mathrm{mK}$ as lowest measuring temperature, which enabled us to resolve dHvA oscillations down to $1.4 \mathrm{~T}$. High-quality single crystals were grown by the usual electrochemical method at Argonne National Laboratory. ${ }^{6}$ The torque dHvA data were obtained at the Grenoble High Magnetic Field Laboratory by means of a homemade $\mathrm{CuBe}$ capacitance cantilever magnetometer mounted on a rotatable sample holder. The capacitance was measured by lock-in technique by use of a capacitance bridge. The intentionally chosen highly sensitive torquemeter allowed us to resolve dHvA oscillations down to rather low fields $(\sim 1.7 \mathrm{~T})$ but, on the other side, resulted in nonlinearities for large torque signals as occurring for the superconducting hysteresis loops at low fields and at higher fields caused by the huge oscillation amplitudes. Since the torque is given by $\vec{\tau}=\vec{M} \times \vec{B}$, where the anisotropic magnetization $\vec{M}$ of the sample is mainly oriented normal to the highly 


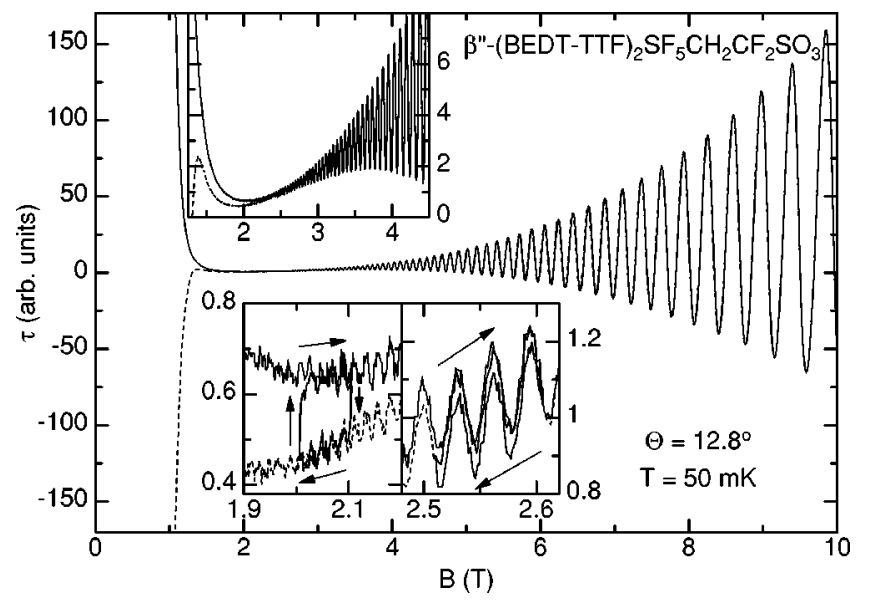

FIG. 1. Field dependence of the torque signal for up (solid line) and down sweep (dashed line). The insets show enlargements of the low-field region visualizing the irreversible magnetization. In the lower insets two hysteresis loops at $2-2.1 \mathrm{~T}$ and $2.5-2.6 \mathrm{~T}$ are included.

conducting BEDT-TTF layers, large $\tau$ resulted especially at higher angle $\Theta$, where $\Theta$ is the angle between $B$ and the normal to the conducting layers. We therefore restricted the field range of the torque data discussed here to fields below $10 \mathrm{~T}$, although the magnet allowed to reach fields up to $28 \mathrm{~T}$.

The dHvA data gathered by use of the field-modulation technique were obtained in a dilution refrigerator equipped with a superconducting 13.6 T magnet at Bristol. Special care was taken to optimize the sensitivity of the pick-up coil system. Sinusoidal modulation fields with amplitude $b_{0}$ from 7 to $30 \mathrm{mT}$ and frequencies $\nu$ of 5.2 and $36.2 \mathrm{~Hz}$ were applied. The signal was detected on the second harmonic $2 \nu$ by lock-in technique. Thereby, the dHvA signal is modulated by the Bessel function of second order with argument $2 \pi b_{0} F / B^{2}$, where $F$ is the dHvA frequency which in our case is $198 \mathrm{~T}$ for $\Theta=0$ and which is growing with $1 / \cos (\Theta)$. In previous experiments, the perfect two dimensionality of the electronic band structure was proven by the sawtooth shape of the dHvA oscillations, which agrees surprisingly well with the signal expected for a two-dimensional (2D) metal with fixed chemical potential. ${ }^{7}$

One important superconducting parameter which can be determined only with large uncertainty for many organic superconductors is the upper critical field, $B_{c 2}$ for $T \rightarrow 0$. The increasingly weak and broad anomalies in bulk experimental methods prevent the extraction of precise values. In the present case, specific-heat data below about $1 \mathrm{~T}$ may be extrapolated to $B_{c 2} \approx 3.6 \mathrm{~T}$. ${ }^{2,5}$ This is consistent with torquemagnetization data at $20 \mathrm{mK}$ which reveal a somewhat lower $B_{i r r} \approx 3.4 \mathrm{~T}$. ${ }^{2}$ Figure 1 shows recent torque data for up and down field sweeps between 0 and $10 \mathrm{~T}$. The angle $\Theta$ $=12.8^{\circ}$ was chosen because of the high sensitivity of the torquemeter for the superconducting and oscillating signal at this orientation. Thereby, $B_{c 2}, F$, and the effective mass increase only slightly (by $2.5 \%$ ) compared to $\Theta=0^{\circ}$. The huge hysteresis at lower fields - saturating the torquemeterdiminishes only very gradually towards higher fields as visualized by the insets of Fig. 1. The irreversible region pre-

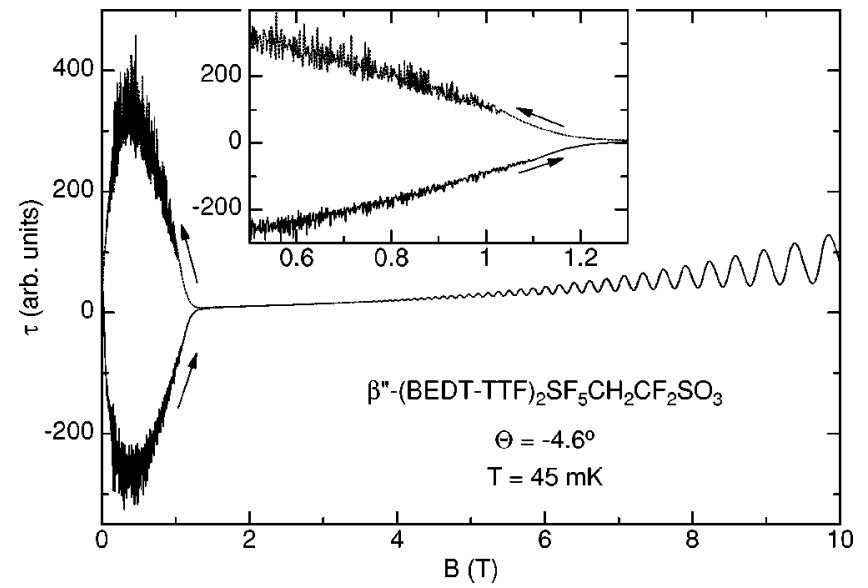

FIG. 2. Field-dependent torque signal for up and down sweeps at $\Theta=-4.6^{\circ}$. At low fields, the noisy magnetization signal is due to flux jumps in the sample, which seize clearly inside the hysteresis loop (inset).

vails up to about $3.5 \mathrm{~T}$ as was verified by measuring small hysteresis loops in a second run (not shown). Thereby, the up sweep was stopped followed by a small $B$ reduction after which the up sweep was continued. As can be seen from the lower insets of Fig. 1, the signals obtained from these small loops lie perfectly on top of the main up and down sweeps. This verifies the existence of superconductivity up to at least $3.5 \mathrm{~T}$ at $50 \mathrm{mK}$ and proves the stability of the used torquemeter. These kind of hysteresis loops have been measured for many different angles up to $\Theta=90^{\circ}$ for which hysteretic behavior was found up to about 19.5 T (data not shown).

At smaller $|\Theta|$, complete hysteresis loops could be resolved without overloading the torquemeter (Fig. 2). At low fields and dilution-refrigerator temperatures, pronounced magnetization spikes appeared (inset of Fig. 2). These abrupt magnetization changes have been found previously in organic $^{8,9}$ and cuprate ${ }^{10}$ superconductors and are caused by a sudden reorganization of magnetic flux entering or leaving the sample. As discussed in more detail in Ref. 9, the thermal link of the sample to the surrounding cryogenic bath becomes increasingly worse towards very low temperatures leading to thermal instabilities with parts of the sample becoming metallic for a short time. This allows magnetic flux to quickly enter or leave the sample leading to slightly different metastable vortex arrangements. Since these flux jumps occur only when vortex pinning is strong, their occurrence indicates the existence of a vortex solid. ${ }^{9}$ As previously observed for the organic superconductor $\kappa-(\mathrm{ET})_{2} \mathrm{Cu}(\mathrm{NCS})_{2}$, the flux jumps suddenly disappear clearly below $B_{i r r} \cdot{ }^{9}$ This can be taken as an indication for a quantum-melting transition from a $2 \mathrm{D}$ vortex-lattice phase to a quantumliquid phase. In the present case, for $\beta^{\prime \prime}$-(BEDT-TTF $)_{2} \mathrm{SF}_{5} \mathrm{CH}_{2} \mathrm{CF}_{2} \mathrm{SO}_{3}$, this latter phase seems to extend over a rather broad field range. However, a more detailed investigation of the vortex-phase diagram still has to be performed for this material.

By use of the field-modulation technique, dHvA signals $M_{2 f}$ like that shown in Fig. 3 were obtained after dividing the raw signal by the Bessel function of second order, 


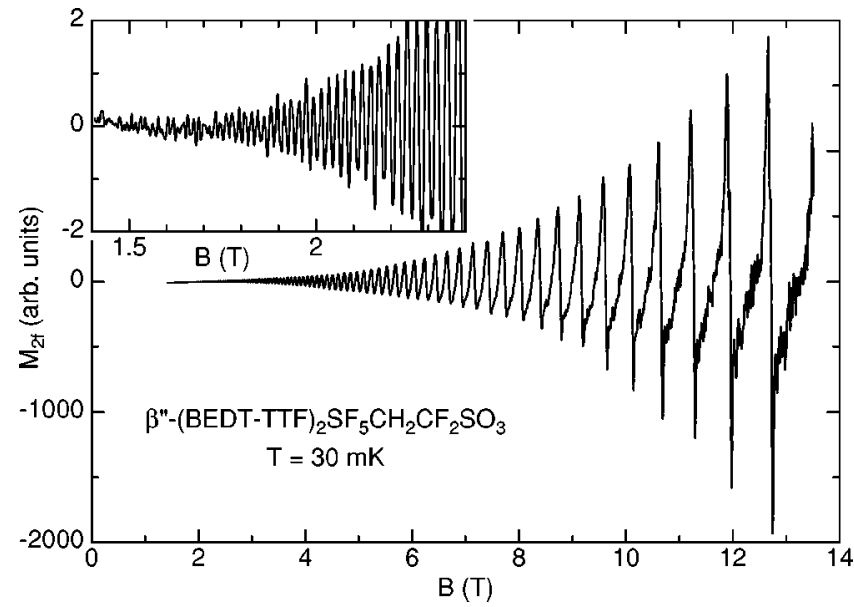

FIG. 3. dHvA signal as measured by the modulation-field technique and corrected by the Bessel function (see text). The inset shows the low-field part with resolvable dHvA oscillations down to $1.4 \mathrm{~T}$.

$J_{2}\left(2 \pi b_{0} F / B^{2}\right)$. At high fields, the dHvA signal develops a large harmonic content with sharp spikes and steep slopes. This signal shape reflects the 2D nature of the electronic systems and can be understood from the known sawtooth shape of the dc magnetization $M{ }^{7}$ For small modulation-field amplitudes $b_{0}$, the modulation-field signal detected here (second harmonic) is proportional to $B^{4} d^{2} M / d B^{2} .{ }^{4}$ Indeed, when comparing $M_{2 f}$ with $B^{2} d^{2} \tau / d B^{2}$ obtained from torque dHvA data of another sample, ${ }^{7}$ excellent agreement between both signals was found (data not shown).

The most important and unexpected result of the present investigation is the field dependence of the dHvA signal in the superconducting state. dHvA oscillations down to about 1.4 T, i.e., $B / B_{c 2}<0.4$, could be detected (inset of Fig. 3). For a detailed analysis of the $\mathrm{dHvA}$ and $\mathrm{SdH}$ amplitudes, we performed Fourier transformations over five oscillations shifting the field interval (which is constant in $1 / B$ ) by one oscillation successively. ${ }^{11}$ The resulting amplitudes $A_{F T}$ for the modulation-field data presented in Fig. 3, torque data close to $\Theta=0^{\circ}$ (not shown), and previously measured $\mathrm{SdH}$ data $^{12}$ are shown in the so-called Dingle plot for a $2 \mathrm{D}$ metal (Fig. 4). Thereby, $A_{F T} B \sinh (X) T^{-1}$ for the modulation-field data $\left[A_{F T} \sinh (X) T^{-1}\right.$ for the torque dHvA and for the $\mathrm{SdH}$ data] is plotted on a logarithmic scale as a function of $1 / B$, with $X=2 \pi^{2} k_{B} m_{c} T / e \hbar B$ where $m_{c}$ is the effective cyclotron mass obtained from the temperature dependence of the oscillation amplitudes. ${ }^{4}$ In the normal state of a 2D metal, straight lines are expected for this kind of plot. For all data sets obtained by the three independent measuring methods, clear concave curvatures are evident at higher fields. Above about $6 \mathrm{~T}(1 / B<0.167)$, the data reside reasonably well on a straight line. We, therefore, restricted the fit range describing the normal-state behavior to $B>6 \mathrm{~T}$ and obtained the shown fit lines corresponding to Dingle temperatures of $T_{D}$ $=0.58 \mathrm{~K}$ for the modulation-field data, $T_{D}=0.76 \mathrm{~K}$ for the torque data, and $T_{D}=0.45 \mathrm{~K}$ for the $\mathrm{SdH}$ data. ${ }^{13}$

Our result shows that in the present material, a reduced attenuation of the oscillating amplitude occurs when entering

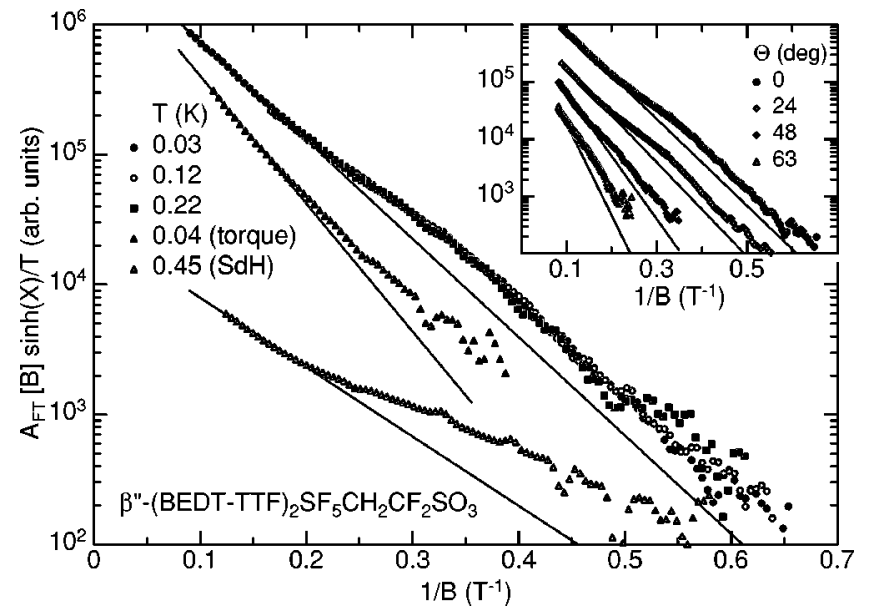

FIG. 4. Two-dimensional Dingle plot of modulation-field data for different temperatures, for torque data $(T=40 \mathrm{mK})$, and $\mathrm{SdH}$ data $(T=0.45 \mathrm{~K})$, all measured at $\Theta \approx 0^{\circ}$. The solid lines are fits to the data above $6 \mathrm{~T}$. The inset shows a Dingle plot of modulationfield data at $30 \mathrm{mK}$ for different angles. The solid fit lines in the inset correspond to an angle-independent Dingle temperature of $0.58 \mathrm{~K}$.

the superconducting state. This behavior is found for different angles (inset of Fig. 4) and for all techniques used to detect magnetic quantum oscillations. Starting from high fields, the first clear deviation of the data from the normalstate Dingle line happens below about $5 \mathrm{~T}$. This is considerably higher than $B_{c 2} \approx 3.6 \mathrm{~T}$ estimated from specific heat. ${ }^{5}$ Although this value has a large uncertainty, the extended influence of superconductivity on the oscillating signal adds another piece of evidence to the importance of superconducting fluctuations in organic metals at high fields. The relevance of these fluctuations has recently been reported for the organic superconductor $\kappa-(\mathrm{ET})_{2} \mathrm{Cu}(\mathrm{NCS})_{2}$ as well. Thereby, $B_{c 2}$ has been treated as a crossover field. ${ }^{14}$ In this latter material, however, an additional attenuation of the dHvA signal is observed, contrary to the present finding.

The reduced attenuation of the magnetic quantum oscillations in the superconducting state is in stark contrast to the usually expected behavior and to previously reported dependences. ${ }^{1}$ Since the predicted additional attenuation of the dHvA signal is mainly caused by the opening of the superconducting energy gap, one might speculate on a gapless, possibly unconventional, superconducting state, which, of course, could only explain the absence of an additional attenuation, but not the reduced attenuation. Specific-heat data, however, prove the fully gapped superconducting state at $B=0$ in this and other organic superconductors. ${ }^{5,15}$ This is expected to become different in a field region close to $B_{c 2}$ where it has been shown that Landau quantization results in gapless quasiparticle excitations. ${ }^{16}$ For the $2 \mathrm{D}$ metal $\beta^{\prime \prime}$-(BEDT-TTF $)_{2} \mathrm{SF}_{5} \mathrm{CH}_{2} \mathrm{CF}_{2} \mathrm{SO}_{3}$, this field region can be estimated to be about $0.5 \mathrm{~T}$ wide. Nevertheless, this cannot explain the reduced attenuation evident for the present superconductor.

Some possible reasons for an enlarged dHvA amplitude in the superconducting state are the following: (i) a reduced effective cyclotron mass in the superconducting state, (ii) a 
reduced quasiparticle scattering rate $\tau^{-1}$, or (iii) a second contribution adding to the measured magnetic quantum oscillations. ${ }^{17}$ In order to check for point (i), we carefully measured the dHvA signal $M_{2 f}$ for different temperatures up to about $1.6 \mathrm{~K}$. By fitting the usual damping factor $R_{T}$ $=X / \sinh (X)$ to the temperature-dependent dHvA amplitudes we obtain, however, a field-independent effective mass $m_{c}$. That is, $m_{c} / m_{e}=2.07(5)$ at $B=3.1 \mathrm{~T}$ (where dHvA oscillations are visible up to $0.54 \mathrm{~K}$ ) and $m_{c} / m_{e}=1.99(5)$ at $B$ $=11 \mathrm{~T}$. Therefore, possibility (i) can be excluded.

Connected with point (ii), a strong increase of the quasiparticle scattering time $\tau$ has been observed for the cuprate superconductors. ${ }^{18}$ For the organic superconductors, on the other hand, such a scenario has not been favored. Microwave data rather suggest a conductivity increase in the mixed state as expected from BCS theory. ${ }^{19}$ Further on, the observed strong increase of the thermal conductivity below $T_{c}$ is usually associated with a reduced scattering of the phonons by quasiparticles. ${ }^{20}$ Nevertheless, a reduced $\tau^{-1}$ in the superconducting state cannot be excluded, especially because above kind of measurements have not yet been performed for the present material.

The final possibility (iii) which might account for the observed effect is the occurrence of additional magnetic quantum oscillations in the mixed state. Indeed, strong quantum oscillations of the superconducting order parameter have been predicted. ${ }^{21}$ However, the resulting magnetization oscillation was predicted to be in antiphase with the normal-state oscillatory contribution leading to the ubiquitous additional damping of the dHvA signal in the mixed state. Yet, to our knowledge, there is no a priori reason against an additional in-phase contribution.

In conclusion, we have presented experimental evidence for enhanced magnetic quantum oscillations in the mixed state of an organic superconductor. The usually observed and expected additional damping of the oscillation amplitude below $B_{c 2}$ is absent or at least much less dominant than for other type-II superconductors. This additional damping might be more than compensated by a reduced quasiparticle scattering or an additional oscillatory contribution in the mixed state.

Part of this work was supported by the ESF Scientific Program on Fermi-liquid instabilities in correlated metals (FERLIN), by the DFG, and by the TMR program under contract No. ERBFMGECT950077. Work at Argonne National Laboratory was supported by the U.S. Deptartment of Energy (Grant No. W-31-109-ENG-38). Work at Portland State University was supported by NSF (Grant No. Che9904316) and the Petroleum Research Fund (ACS-PRF Grant No. 34624-AC7).
${ }^{1}$ T.J.B.M. Janssen et al., Phys. Rev. B 57, 11698 (1998).

${ }^{2}$ J. Wosnitza et al., Phys. Rev. B 62, R11 973 (2000).

${ }^{3}$ K. Yasui and T. Kita, J. Phys. Soc. Jpn. 70, 2852 (2001).

${ }^{4}$ D. Shoenberg, Magnetic Oscillations in Metals (Cambridge University Press, Cambridge 1984).

${ }^{5}$ S. Wanka et al., Phys. Rev. B 57, 3084 (1998). This specific-heat data led to the bulk $T_{c}=4.4 \mathrm{~K}$. The diamagnetic onset is at 5.2 K (Ref. 6).

${ }^{6}$ U. Geiser et al., J. Am. Chem. Soc. 118, 9996 (1996).

${ }^{7}$ J. Wosnitza et al., Phys. Rev. B 61, 7383 (2000); J. Hagel et al., Synth. Met. 120, 813 (2001).

${ }^{8}$ A.G. Swanson et al., Solid State Commun. 73, 353 (1990); T. Sasaki et al., Phys. Rev. B 57, 10889 (1998).

${ }^{9}$ M.M. Mola et al., Phys. Rev. Lett. 86, 2130 (2001); M.M. Mola et al., Int. J. Mod. Phys. B 15, 3353 (2001).

${ }^{10}$ L. Legrand et al., Physica C 211, 239 (1993).

${ }^{11}$ This kind of analysis is not crucial. Other algorithms yield very similar field dependences of the dHvA amplitudes.

${ }^{12}$ The $\mathrm{SdH}$ amplitudes $A_{F T}$ are obtained by reanalyzing the $\mathrm{SdH}$ signal of sample $A$ in Ref. 2.

${ }^{13}$ Strictly speaking, for the determination of $T_{D}$ the band mass $m_{b}$ should be used. For simplicity, we used $m_{c}$ as done by most researchers.

${ }^{14}$ N.J. Clayton et al., Phys. Rev. B 65, 064515 (2002).

${ }^{15}$ H. Elsinger et al., Phys. Rev. Lett. 84, 6098 (2000); J. Müller et al., Phys. Rev. B 65, 140509 (2002).

${ }^{16}$ V.P. Mineev, Physica B 259-261, 1072 (1999); T. Champel and V.P. Mineev, Philos. Mag. B 81, 55 (2001).

${ }^{17}$ A possible change of the $g$ factor would also not explain the observation, since for the present material the spin-splitting factor is almost maximal at $\Theta=0$.

${ }^{18}$ K. Krishana et al., Phys. Rev. Lett. 75, 3529 (1995) and references therein.

${ }^{19}$ M. Dressel et al., Phys. Rev. B 50, 13603 (1994).

${ }^{20}$ S. Belin et al., Phys. Rev. Lett. 81, 4728 (1998); J. Wosnitza et al., Synth. Met. (to be published).

${ }^{21}$ T. Maniv et al., Phys. Rev. B 46, 8360 (1992). 